\title{
Correction to: Role of Bruton's tyrosine kinase in B cells and malignancies
}

Simar Pal Singh ${ }^{1,2,3}$, Floris Dammeijer ${ }^{1,3,4}$ and Rudi W. Hendriks ${ }^{* *}$

\section{Correction to: Mol Cancer \\ https://doi.org/10.1186/s12943-018-0779-z}

Following publication of the original article [1], the authors reported an error in Table 1. Incorrect value was placed under Efficacy (column), R/R non-GCB DLBCL (row).The value $92 \%$ was captured instead of $35 \%$. Corrected table is shown below. The authors would like to apologize for this error.

\begin{abstract}
Author details
'Department of Pulmonary Medicine, Room Ee2251a, Erasmus MC Rotterdam, PO Box 2040, NL 3000, CA, Rotterdam, The Netherlands. ${ }^{2}$ Department of Immunology, Rotterdam, The Netherlands. ${ }^{3}$ Post graduate school Molecular Medicine, Rotterdam, The Netherlands. ${ }^{4}$ Erasmus MC

Cancer Institute, Erasmus MC, Rotterdam, The Netherlands.
\end{abstract}

Published online: 03 April 2019

\section{Reference}

1. Singh PS, Dammeijer F, Hendriks RW. Role of Bruton's tyrosine kinase in B

cellsand malignancies. Mol Cancer. 2018;17:57 https://doi.org/10.1186/

s12943-018-0779-z

\footnotetext{
*Correspondence: r.hendriks@erasmusmc.nl

'Department of Pulmonary Medicine, Room Ee2251a, Erasmus MC

Rotterdam, PO Box 2040, NL 3000, CA, Rotterdam, The Netherlands

Full list of author information is available at the end of the article
} 
Table 1 Clinical trials with BTK inhibitors in B cell malignancies

\begin{tabular}{|c|c|c|c|c|}
\hline Patient population & Therapeutic regimen & Phase & Efficacy & Ref \\
\hline$\overline{\mathrm{R} / \mathrm{R} C L L}$ & Ibrutinib & $\mathrm{Ib} / \mathrm{II}$ & ORR (71\%), PR (20\%) & [11] \\
\hline R/R CLL & Ibrutinib & III & ORR (63\%) & {$[248]$} \\
\hline TN CLL & Ibrutinib & $\mathrm{Ib} / \mathrm{ll}$ & ORR (85\%), CR (26\%) & [199] \\
\hline TN CLL & Ibrutinib & III & ORR (86\%), CR (4\%) & [13] \\
\hline $\mathrm{R} / \mathrm{R} M C L$ & Ibrutinib & $\|$ & ORR (68\%), CR (21\%) & [187] \\
\hline $\mathrm{R} / \mathrm{R} M C L$ & Ibrutinib & III & ORR (72\%), CR (19\%) & [249] \\
\hline R/R WM & Ibrutinib & $\|$ & ORR (91\%), Major response (73\%) & [188] \\
\hline R/R ABC-DLBCL & Ibrutinib & $\|$ & ORR (37\%) & [196] \\
\hline $\mathrm{R} / \mathrm{R} C L \mathrm{~L}$ & Ibrutinib-Rituximab & $\|$ & ORR (95\%), CR (8\%) & [250] \\
\hline $\mathrm{R} / \mathrm{R} C L \mathrm{~L}$ & Ibrutinib-bendamustine-rituximab & III & ORR (83\%), CR (10\%) & [251] \\
\hline $\mathrm{R} / \mathrm{R} \mathrm{MCL}$ & Ibrutinib-Rituximab & $\|$ & ORR (88\%), CR (44\%), PR (44\%) & {$[252]$} \\
\hline R/R CLL & Acalabrutinib & $|/| \mid$ & ORR (95\%) & {$[12]$} \\
\hline$R / R$ & Acalabrutinib & $\|$ & ORR (81\%), CR (40\%), PR (41\%) & [219] \\
\hline R/R CLL & ONO/GS-4059 & I & ORR (96\%) & [222] \\
\hline $\mathrm{R} / \mathrm{R} M C L$ & ONO/GS-4059 & 1 & ORR (92\%) & [222] \\
\hline R/R non-GCB DLBCL & ONO/GS-4059 & I & ORR (35\%) & {$[222]$} \\
\hline R/R CLL & BGB-3111 & 1 & ORR (90\%) & {$[221,253$} \\
\hline $\mathrm{R} / \mathrm{R} \mathrm{MCL}$ & BGB-3111 & I & ORR (80\%) & [253] \\
\hline R/R MZL & Ibrutinib & $\|$ & ORR (51\%) & [254] \\
\hline $\mathrm{R} / \mathrm{R} F \mathrm{~L}$ & Ibrutinib & I & ORR (38\%) & [186] \\
\hline
\end{tabular}

CLL Chronic Lymphocytic leukemia, MCL Mantle cell lymphoma, WM Waldenström's Macroglobulinemia, $A B C-D L B C L$ Activated B-cell Diffuse large B cell Lymphoma, MZL Marginal zone lymphoma, FL Follicular lymphoma, $R / R$ relapsed or refractory, $T N$ treatment-naïve, $O R R$ overall response rate, $C R$ complete response, $P R$ partial response, Major response: complete response or at least $50 \%$ reduction in serum IgM levels 